\title{
Effects of Literacy in Early Visual and Occipitotemporal Areas of Chinese and French Readers
}

\author{
Marcin Szwed $^{1,2,3}$, Emilie Qiao ${ }^{2,3,4}$, Antoinette Jobert ${ }^{2,5}$, \\ Stanislas Dehaene ${ }^{2,5,6,7}$, and Laurent Cohen ${ }^{3,4,8}$
}

\begin{abstract}
How does reading expertise change the visual system? Here, we explored whether the visual system could develop dedicated perceptual mechanisms in early and intermediate visual cortex under the pressure for fast processing that is particularly strong in reading. We compared fMRI activations in Chinese participants with limited knowledge of French and in French participants with no knowledge of Chinese, exploiting these doubly dissociated reading skills as a tool to study the neural correlates of visual expertise. All participants viewed the same stimuli: words in both languages and matched visual controls, presented at a fast rate comparable with fluent reading. In the Visual Word Form Area, all participants showed enhanced responses to their known scripts. However, group differences were found in occipital cortex. In French readers reading French, activations were enhanced in left-hemisphere visual area V1, with the strongest differences between French words
\end{abstract}

\section{INTRODUCTION}

Humans have developed a variety of scripts. Some, like the alphabetic Latin script, use a small set of relatively simple letters. Other, such as the logographic Chinese script, use a large $(>1000)$ set of complex logographs. In this article, we explore how the visual system adapts to the particular demands posed by these two very different scripts.

Most previous studies focused on word recognition in higher visual and general language areas (reviewed in Wu, Ho, \& Chen, 2012; Perfetti, Nelson, Liu, Fiez, \& Tan, 2010; Chen, Xue, Mei, Chen, \& Dong, 2009; Bolger, Perfetti, \& Schneider, 2005; Tan, Laird, Li, \& Fox, 2005). Here, we focused primarily on written word recognition at the level of early and intermediate visual cortices. Mature readers can read extremely fast, up to 200 words

${ }^{1}$ Jagiellonian University, Krakow, Poland, ${ }^{2}$ INSERM, Gif sur Yvette, France, ${ }^{3}$ INSERM, Paris, France, ${ }^{4}$ Université Pierre-et-Marie-Curie, ${ }^{5}$ Commissariat à l'Energie Atomique, Institute of Bioimaging, Neurospin, Gif sur Yvette, France, ${ }^{6}$ Collège de France, ${ }^{7}$ Université Paris, ${ }^{8}$ Hôpital de la Salpêtrière, Paris, France

and their controls found at the central and horizontal meridian representations. Chinese participants, who were not expert French readers, did not show these early visual activations. In contrast, Chinese readers reading Chinese showed enhanced activations in intermediate visual areas $\mathrm{V} 3 \mathrm{v} / \mathrm{hV} 4$, absent in French participants. Together with our previous findings [Szwed, M., Dehaene, S., Kleinschmidt, A., Eger, E., Valabregue, R., Amadon, A., et al. Specialization for written words over objects in the visual cortex. Neuroimage, 56, 330-344, 2011], our results suggest that the effects of extensive practice can be found at the lowest levels of the visual system. They also reveal their cross-script variability: Alphabetic reading involves enhanced engagement of central and right meridian V1 representations that are particularly used in left-to-right reading, whereas Chinese characters put greater emphasis on intermediate visual areas.

per minute. Recently, two experiments (Szwed et al., 2011; Dehaene et al., 2010) have demonstrated that learning to read an alphabetic script involves the acquisition of visual expertise for letters in early visual areas V1/ V2, probably due to the necessity for fast, parallel recognition of letter strings. In those experiments, we observed enhanced activation to words relative to well-matched scrambled stimuli during reading, which might have reflected massive low-level processing that allows fast and parallel integration of letter features. Under this view, those efficient early visual processing mechanisms contribute to make reading so effortless.

Relative to this earlier work, the present research had two main objectives. First, we probed the existence of such an early visual enhanced activation for written characters in an experiment where expert readers were compared with nonexperts. Our prediction was that these activations should be present only in expert readers. Second, we sought to determine whether the early visual areas of Chinese readers show signs of reading expertise similar to those found in readers of the Latin alphabet, or whether there are some cross-script differences due to differences in orthographic structure and visual appearance of these two scripts. 
To properly study the function of early and intermediate areas in reading, one needs rigorous control conditions, because any visual stimulus, even devoid of contours or shapes such as white noise, will activate early and intermediate visual areas (e.g., Grill-Spector, Kushnir, Hendler, \& Malach, 2000). Most previous studies used either fixation or other stimuli poorly matched in visual features such as faces (however, see Ben-Shachar, Dougherty, Deutsch, \& Wandell, 2007; Uchida et al., 1999). In the current study, we used carefully matched scrambled visual controls that had the same amount of contrast, line length, surface area, and envelope shape, similar to Szwed and colleagues (2011). Also, unlike in most previous studies, French and Chinese words were presented at a fast presentation rate close to everyday, fast reading conditions (150 words per minute).

Here, the same stimuli (written French words and nonwords, written Chinese words, objects, and respective scrambled controls) were presented to both French and Chinese college students (Figure 1). Both French and Chinese participants were fluent readers in their native scripts. Chinese participants had arrived in France less than 2 years before the experiment and were not proficient readers of French. Conversely, French participants had no knowledge of Chinese. The two participant groups' reading skills were thus partially dissociated, although the dissociation was not perfect as Chinese participants could read some French words. This dissociation was used as a tool to study the neural correlates of reading expertise.

\section{METHODS}

\section{Stimuli}

Stimuli used (Figure 1A) were printed Chinese and French words, nonwords derived from French words, scrambled Chinese and French words, objects, and scrambled objects.

We used 46 French nouns, six to eight letters long with median frequency (New, Pallier, Brysbaert, \& Ferrand, 2004) of 4.3 per million and 46 two-character Chinese nouns with median frequency (Cai \& Brysbaert, 2010) of 4.6 per million [frequency difference nonsignificant, $t(77)=-0.46 ; p=.65]$. Chinese words subtended a visual angle of $2.4 \times 1^{\circ}$. French words subtended a more elongated field of up to $4.8 \times 0.68^{\circ}$ (median length $=4^{\circ}$, constant height $=0.68^{\circ}$ ). Objects subtended more varied visual angles of up to $5.4 \times 2^{\circ}\left(\right.$ median $\left.=4 \times 0.8^{\circ}\right)$. We chose a line width and font size allowing us to match satisfactorily overall line length and luminance between French and Chinese words. French and Chinese words were also matched in number of vertex features (roughly corresponding to line junctions; for details, see Szwed, Cohen, Qiao, \& Dehaene, 2009). This meant choosing Chinese words made out of complex (e.g., 歉) rather than simple characters (e.g., 干). This choice also allowed us to avoid Chinese characters that could resemble alphabet letters (e.g., 己). Compared with Latin letters, these Chinese characters had a much higher perimetric complexity (Pelli, Burns, Farell, \& Moore-Page, 2006). Unpronounceable French nonwords (e.g., "GSDBIIUO") were made by rearranging the letters of French words used in the experiment (e.g., "BIGOUDIS") into letter strings that had the lowest possible resemblance to real words (lowest possible average letter bigram frequencies computed with the Lexique word frequency database; New et al., 2004).

Scrambled words and objects were made by randomly shuffling individual features while maintaining the same stimulus surface and envelope (Szwed et al., 2011). To allow shuffling of the characters' individual features, small gaps of the size of one pixel were made at the line junctions of individual characters (Figure 1A). The main differences between intact and scrambled words were twofold. First, individual characters in French and Chinese words were separated by fixed spaces of $<0.2^{\circ}$; the scrambling procedure removed this spacing. Second, in intact words, contours were present in recurring positions (e.g., top parts of $\mathrm{T}, \mathrm{E}$, 支, 来, ...); in scrambled stimuli, the location of contours was random.

Objects were made by rearranging individual features of a French word into an object: for example, the car depicted in the figure was made out of the features of the word "BIGOUDIS." We attempted to keep the overall stimulus envelope horizontal and roughly similar to words (see examples in Figure 1A, C and averages in Supplemental Figure 1B). Recognition of objects was tested in a pilot naming task in which participants saw the objects for $200 \mathrm{msec}$. The average recognition rate was 77\% correct ( $n=8$ participants).

\section{Participants}

The participants were 14 right-handed, native French speakers, 20-31 years old (nine women, five men), and 11 right-handed native Chinese speakers, 20-30 years old (seven women, four men) who came to France less than 2 years before the experiment was carried out. All participants were college students or graduates, and all gave written informed consent to participate in the present fMRI study. They had no history of neurological or psychiatric disease. Their vision was normal or corrected to normal. The project was approved by the regional ethical committee.

\section{Structure of the Experiment}

Before the main fMRI experiment, participants were presented with the same 46 French and Chinese words that they were about to see in the imaging session. These words were displayed for $200 \mathrm{msec}$, a duration identical to the one used in the fMRI experiment. Participants were asked to read the words aloud. 
Figure 1. Design and behavioral results. (A) Stimuli consisted of objects, Chinese words, French words, French nonwords, and their scrambled counterparts. Scrambled words and objects were made by randomly shuffling individual features while maintaining the same stimulus surface area and envelope shape. Unpronounceable nonwords (e.g., "GSDBIIUO") were made by rearranging the letters of French words used in the experiment (e.g., "BIGOUDIS") into letter strings that had the lowest possible orthographic plausibility (lowest possible average letter bigram frequency). Objects were made by rearranging the features of a word into the shape of an object: for example, the car depicted in the figure is made out of the features of the word "BIGOUDIS." (B) Each fMRI scanning session included 12 blocks for each stimulus type. Blocks were separated by fixation periods that lasted either 3, 4, or 5 sec. (C) In each block, 13 stimuli of one given type were displayed for 200 msec separated by a 200-msec fixation screen (5 sec total block duration, 150 stimuli per minute). (D) Before the fMRI experiment, participants were presented with the same 46 French and Chinese words that they were about to see in the imaging session. Words were presented for $200 \mathrm{msec}$, a duration identical to the one used in the fMRI experiment. Participants were asked to read the words aloud. Both French and Chinese participants were near $100 \%$ correct for their native scripts. Chinese participants could also partially recognize French words

(55\% average accuracy, individual results represented by dots). (E) During the fMRI scanning sessions, participants were asked to press a button whenever they saw a group of circles (oddball detection task). RTs did not differ across participant groups $(p=.50)$, with no stimulus $\times$ group interaction $(p=.44)$. We conclude there was no difference in task set across participant groups. Error bars in (E) represent the SEM.
The main fMRI experiment contained a series of short blocks of rapid sequential stimuli (similar to Vinckier et al., 2007; Figure 1B) designed to minimize top-down effects by interrupting the late phase of word processing taking place in the language network, the source of those top-down effects (e.g., Marinkovic et al., 2003). Each block contained stimuli of one of the seven possible types (Figure 1A). In each block, 13 such stimuli were

\section{Design and behavioral results}

French

Objects

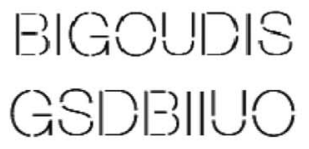

scrambled

French words

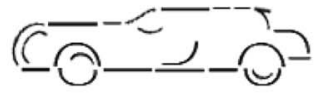

\section{scrambled} objects
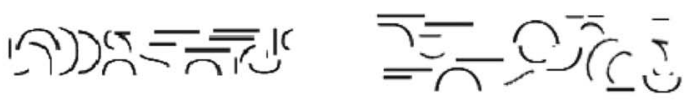

words \& nonwords made out of French word fragments

B

C

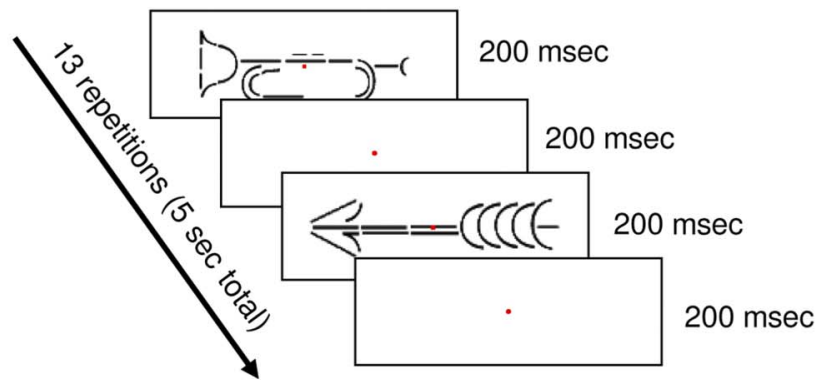

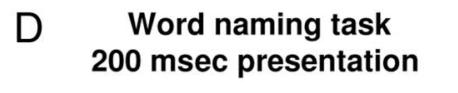

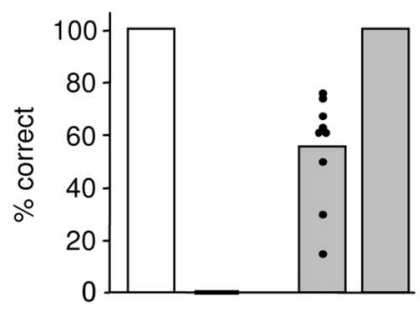

words: french chinese french chinese

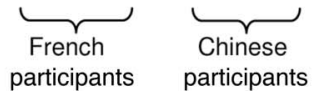

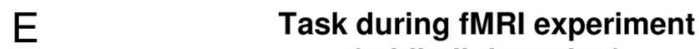
(oddball detection)

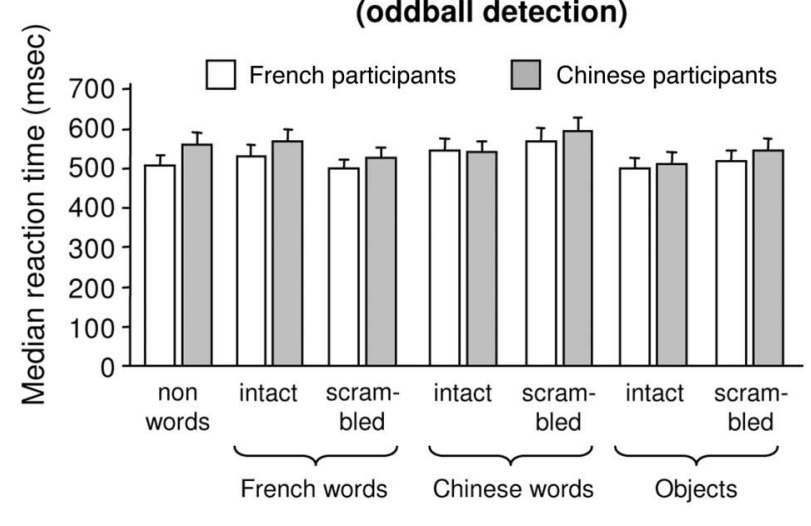


the blocks, one stimulus was replaced with a target-a set of six circles to which participants were instructed to respond by pressing a button with their right hand. The target's envelope shape was matched to the shape of stimuli. For French words, the circles were arranged horizontally, whereas for Chinese words and objects, they were arranged in a diamond-like configuration matched to the overall shape of Chinese and object stimuli.

\section{Stimulation and Acquisition Parameters}

Stimuli were presented in the center of the visual field using the E-prime software (PST, Pittsburgh, PA). We used a 3-T MRI (Siemens Trio TIM; Erlangen, Germany) with a 12-channel head coil, and a gradient EPI sequence sensitive to BOLD contrast ( 40 contiguous axial slices, acquired using ascending interleaved sequence, $3 \mathrm{~mm}$ thickness, $\mathrm{TR}=2400 \mathrm{msec}$, flip angle $=90^{\circ}, \mathrm{TE}=30 \mathrm{msec}$, in-plane resolution $=3 \times 3 \mathrm{~mm}$, matrix $=64 \times 64)$. For each acquisition, the first four volumes were discarded to reach equilibrium. T1-weighted images were also acquired for anatomical localization.

\section{Analysis}

Individual imaging data processing was performed with SPM8 software and included corrections for EPI distortion, slice acquisition time, and motion; normalization to the MNI anatomical template; Gaussian smoothing $(5 \mathrm{~mm}$ FWHM) and fitting with a linear combination of functions derived by convolving a standard hemodynamic response function with the time series of the stimulus categories (defined by stimulus onsets and durations). Each block was treated as a single event, and fixation baseline was modeled implicitly. For whole-brain analysis (Figures 4, 6, and 8), individual contrast images were computed for each stimulus type minus baseline, then smoothed for the second time ( $8 \mathrm{~mm}$ FWHM), and eventually entered in an ANOVA for random effect group analysis. For ROI analyses (Figures 2, 3, and 7) as well as for the singleparticipant examples (Figure 5), we used unsmoothed data. Functional maps were created using the xjview toolbox (www.alivelearn.net/xjview8/). Further analysis was done using Matlab (Matlab Inc., Natick, MA), Minitab (Minitab Inc., State College, PA), and R (www.r-project.org).

Activation values reported in ROI plots are in percentage BOLD activation (beta), which were computed using the MarsBar toolbox. ROI activations were computed relative to the fixation baseline. The between-hemisphere ANOVA results in the ROI results sections always refer to the interaction of stimulus type (intact vs. scrambled) with hemisphere.

For all whole-brain analyses, we used a threshold of $p<.01$ voxel-wise and $p<.05$ cluster-wise false discovery rate (FDR) corrected for multiple comparisons across the whole brain. (The low voxel threshold of $p<.01$ was used to facilitate the detection of relatively weak but extended areas of activation, whereas the cluster-level threshold still ensured correction for the whole-brain search. Similar results were observed at voxel

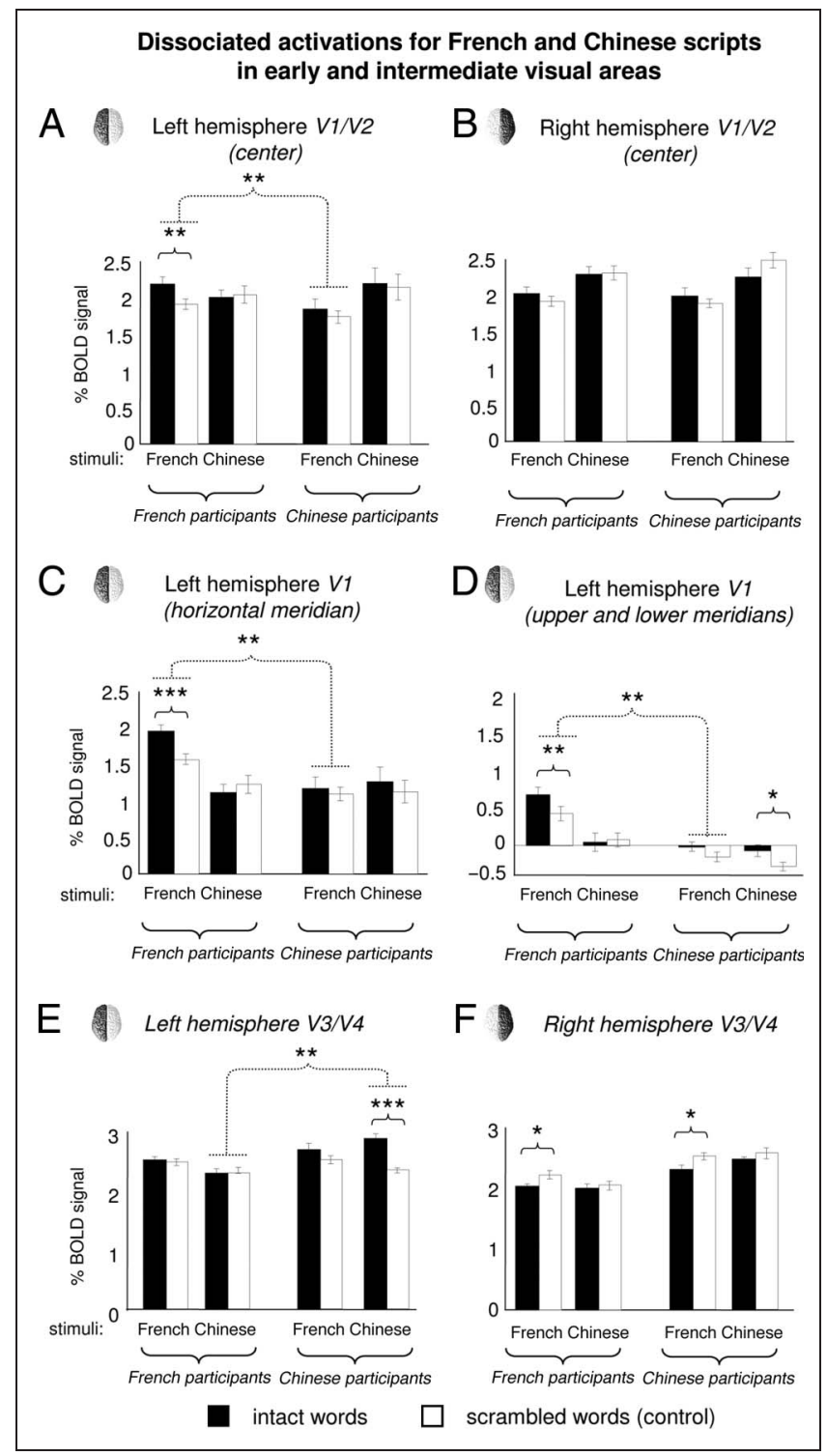

Figure 2. Dissociated activations for French and Chinese scripts in early and intermediate visual areas. Activations are shown for ROIs located in low level visual areas V1/V2 (A-D) and intermediate visual areas V3v/hV4 (E-F). Greater responses to French words relative to scrambled controls were found only in the left-hemisphere early visual areas V1/V2 of French participants. These enhanced responses were strongest at the center and horizontal meridian representations (A, C) but also encompassed the vertical meridian (D). Statistically significant differences between intact and scrambled stimuli are marked with solid brackets. Dotted brackets mark statistically significant interactions between French and Chinese participants (subject group $\times$ entire/scrambled stimulus form). ${ }^{* * *} p<.001$, $* * p<.01, * p<.05$. Activation was computed relative to a fixation baseline. Error bars represent the SEM across participants after subtraction of the individual participants' overall mean, equivalent to the error term in ANOVA with participants as random factor. 
Figure 3. Activations in the fusiform region for French and Chinese participants. Activations are shown for ROIs located in the fusiform region of the high-level visual cortex. Statistically significant differences between intact and scrambled stimuli are marked with solid brackets. Dotted brackets mark statistically significant interactions between French and Chinese participants (subject group $\times$ intact/ scrambled stimulus form). $* * * p<.001, * * p<.01$, $* p<.05$. Activation was computed relative to a fixation baseline. Error bars represent the SEM across participants after subtraction of the individual participants' overall mean, equivalent to the error term in ANOVA with participants as random factor.

$p<.001$, although not necessarily at corrected levels of significance.)

\section{Mapping of Retinotopic Areas and Definition of ROIs}

All ROI analyses were performed on unsmoothed data.

After the main fMRI experiment, retinotopic visual areas were mapped using the meridians method (Claeys et al., 2004). Stimuli consisted of flashing checkerboard wedges covering either the lower vertical meridian, the upper vertical meridian, or both horizontal meridians, as well as flashing checkerboard rings covering the central $\left(2^{\circ}\right)$ region of the visual field. The borders of the retinotopic areas (e.g., between V1 and V2) were localized along the line of highest response to the meridian wedge stimulus. The retinotopic ROIs (Figure 2) were defined, in each individual participant, as the 25 most significant voxels responding (1) to the central ring stimulus for the "center" V1/V2 ROI and V3v/hV4 ROI, (2) to the contrasts "vertical meridian minus center" and "horizontal meridian minus center" for the "vertical meridian" and "horizontal meridian" contrasts, respectively. Because the meridians mapping method used here is not very exact at determining boundaries between adjacent visual areas in the central visual field (Schira, Wade, \& Tyler, 2007), we chose to pool together central representations for V1 and V2 and, separately, for V3v and hV4 (Figure 2). Note that the hV4 fMRI results are inherently contaminated by artifacts from the transverse sinus, which might have influenced our results (Winawer, Horiguchi, Sayres, Amano, \& Wandell, 2010).

Fusiform ROIs were chosen as the 25 most significant voxels responding to the (all stimuli fixation baseline) contrast, within canonical boundaries of the Visual Word
Form Area, that is, between $y=-70$ and $y=-50$ in the ventral occipitotemporal region (MNI -39 - 57-9; Cohen et al., 2002). This particular method was used to select the most relevant fusiform ROIs in each participant by taking account of the interindividual variability. It was also orthogonal to the comparisons being made in this article (e.g., words minus scrambled controls) and thus allowed us to avoid "double dipping" (Vul, Harris, Winkielman, \& Pashler, 2009). Following similar principles, IPS ROIs were selected in each individual participant, as the 25 most significant voxels of the "objects minus scrambled objects" contrast within the anatomically defined intraparietal sulcus (IPS) area.

\section{RESULTS}

\section{Behavioral Results}

Before the fMRI experiment, participants saw the same 46 French and Chinese words that they were about to see during the imaging session. These words were presented for $200 \mathrm{msec}$, a duration identical to the one used in the fMRI experiment. Both Chinese and French participants were $100 \%$ correct at naming words written in their native script (Figure 1D). However, although all Chinese participants were enrolled in French university and read French text on a daily basis, they nonetheless had problems with naming words presented at such a rapid rhythm, with an average recognition rate of $55 \%$. Thus, Chinese participants were not expert French readers. French participants, who had never learned any Chinese, could not name a single Chinese word.

During the main fMRI scanning session, participants performed a simple oddball detection task, which kept their attention focused on the stimuli (see Methods). The 
percentages of correct oddball detections and RTs were analyzed in an ANOVA with Participant Group (Chinese vs. French) as a between-participant factor and Stimulus Type as a within-participant factor. The percentages of correct oddball detections were close to $100 \%$ (mean = $98 \%$ ) and did not differ across groups, $F(1,23)=0.18$, $p=.67$, nor across types of stimuli, $F(6,138)=1.74$, $p=.12$. RTs (Figure 1E) were different across stimulus types, $F(6,138)=7.77, p<.001$. However, we found that they did not differ across participant groups, $F(1,23)=$ $0.46, p=.50$, with no Stimulus Type $\times$ Group interaction, $F(6,138)=0.98, p=.44$. An ANOVA with Stimulus Type modeled as two orthogonal within-participant factors (stimulus category: object/word; and stimulus form: intact/ scrambled) revealed that detecting oddballs among objects yielded shorter RTs than among all other stimuli (24 msec difference, $F(1,23)=13.75, p=.001$ ) and that detecting oddballs among scrambled stimuli tended to give slightly longer RTs than among intact stimuli (9 msec difference, $F(1,23)=5.86, p=.02$ ), with a marginally significant interaction between word/object and scrambled/intact factors, $F(1,23)=3.93, p=.058$. Critically, there were no group effects or interactions involving the group effect (all $p>$ .4 ), allowing us to conclude that there was no difference in task set between Chinese and French participants.

\section{ROI Analysis of Activations to Reading in the Visual System}

The main objectives of the current study were to (1) test the existence of early visual activations for written characters (Szwed et al., 2011) in an experiment where the brain activity in expert readers is compared with brain activity in nonexperts and (2) determine whether early visual areas of Chinese readers show signs of reading expertise similar to those found in Latin alphabet readers or whether there are some cross-script differences.

To this aim, we mapped retinotopic visual areas in each participant (see Methods). We then examined ROIs located in areas V1/V2 and V3v/hV4 (Figure 2). For the sake of clarity, in this section we describe only activations to words and their scrambled controls. Activations to French nonwords and to objects are described in subsequent sections.

\section{V1/V2-Center Representation}

For French words, stronger activations for intact versus scrambled word stimuli were found only in French participants in the left hemisphere (Figure 2A-B; 22\% difference, $p=.005$; interaction with hemisphere: $p=.03$ ). No such difference was found for Chinese participants viewing the same alphabetic stimuli (left and right ROIs, $p>.45$; French-Chinese participant group interaction: $p=.006$ ). Thus, stronger activations for French words compared with the scrambled French words condition were present only in expert French readers and were absent in Chinese participants.

We expected similar markers of expertise for Chinese words in Chinese participants. However, we found that, relative to scrambled controls, Chinese words did not lead to enhanced activations in the left hemispheres of either Chinese or French participants (all $p>.10$ ).

\section{V1-Horizontal Meridian}

At the horizontal meridian representation, stronger activations for intact versus scrambled word stimuli were found, again, only in French participants in the left hemisphere (Figure 2C; 27\% difference, $p<.001$ ). No such difference was found in French participants in the right hemisphere $(p=.13$ ) nor for Chinese participants viewing the same alphabetic stimuli (both hemispheres, $p>.38$ ). The responses to intact French words and their scrambled controls were generally stronger than responses to intact Chinese words and their scrambled controls (both populations, $p<.02$ ) most likely because Latin stimuli overlapped more with the horizontal meridian area than Chinese stimuli (see Figure 1 and Methods).

\section{V1-Vertical Meridian}

Vertical meridian activations (Figure 2D) were generally low, because all stimuli fell outside of this ROI. Indeed, only French participants' responses to French words were significantly stronger than the fixation baseline $(p=.02$; $p>.27$ for other conditions), with the responses to intact stimuli being stronger than responses for scrambled word stimuli (Figure 2D, $p=.002$ ).

\section{Areas $V 3 v / h V 4$}

In this ROI, French words yielded activations that were either similar (left hemisphere, both groups $p>.1$ ) or lower (right hemisphere, both $p<.02$ ) than scrambled controls (Figure 2E-F). However, an important cross-script effect was found for Chinese words. In the left hemisphere, responses to Chinese words were stronger than for scrambled controls, but only in Chinese participants $(p<.001$; Figure 2E) and not in French participants ( $p=.88$; between-group interaction, $p=.003)$. This effect was absent in the right hemisphere, where the activations for Chinese words remained similar to activations for scrambled Chinese words $(p>.29$ for both Chinese and French participants). The effect seen in Chinese participants was also strongly left-predominant as attested by its interaction with hemisphere $(p=.044)$.

The results of the V1/V2 and V3v/hV4 analyses thus show that early and intermediate visual activations observed, respectively, only in French reading French and in Chinese reading Chinese, are dependent on the existence of reading expertise for a given script [Interaction 
Brain Area (center V1/V2 vs. V3/V4) × Participant Group $\times$ Script, $p<.004$ ].

\section{Fusiform}

In addition to retinotopic ROIs, we also analyzed ROIs in the fusiform region.

In the left fusiform area, French words evoked stronger responses than scrambled French words in both Chinese and French populations $(p=.007$ and $p=.001)$. Such differences were absent from the right hemisphere (both $p>$.81). Chinese word activations, in contrast, were more bilateral. Chinese words evoked stronger responses than scrambled Chinese words in the left as well as the right hemispheres of both groups (Chinese: $p=.001 \mathrm{LH}, p=$ $.003 \mathrm{RH}$; French: $p=.002 \mathrm{LH}, p<.001 \mathrm{RH})$. There was a tendency for the difference in activations between intact and scrambled stimuli in Chinese participants to be larger in the left than in the right hemisphere; however, it did not reach statistical threshold (interaction hemisphere/ stimulus, $p=.18)$.

\section{Whole-brain Analysis of Reading in French and Chinese Scripts}

Following this focused ROI analysis, we now move to a whole-brain analysis to describe the spatial extent of activations to words. We also report activations to other stimuli: nonwords and objects.

A list of areas activated by the main conditions is provided in Table 1 . In both French and Chinese participants, reading in ones' native script versus scrambled controls activated a similar network of language-related areas (Figure $4 \mathrm{~A}$ and $\mathrm{D}$; Table 1). The most visible differences between French and Chinese participants were seen in the early and intermediate visual areas. Consistent with the ROI analyses, in French participants, activations for French words were seen in the left fusiform and early visual areas but not in intermediate visual areas (Figure 4A; peak MNI: $-9-93-3, Z=3.43$, dotted circle). In Chinese participants, activations for Chinese words versus scrambled controls were seen in the left fusiform and intermediate visual areas but not in early visual areas (Figure 4D; MNI: $-45-84-6, Z=6.69$, solid circle). Activations to French words versus scrambled control stimuli in two individual French participants are shown in Figure 5.

Consistent with the ROI analysis, Chinese participants showed more right-hemisphere fusiform activations for their native language versus scrambled control than French participants (Figure 4; Chinese MNI: $45-51-21, Z=$ 5.59; French MNI: $57-48-12, Z=3.10$ ), although their left VWFA activations were still stronger and more extended that the corresponding right-hemisphere activations (left hemisphere: $Z>8$; right hemisphere: $Z=5.59$ ).

Chinese participants could also partially recognize French words (Figure 1D). In Chinese participants, French words evoked stronger activations than scrambled words in the fusiform regions at the location of the VWFA, but not in early or intermediate visual areas (Figure 4B; Table 1).

\section{Activations to Chinese Words in Naive French participants}

French participants with no experience of Chinese showed substantial activations for Chinese words (Figure 4C) in the fusiform cortex, lateral occipital area, and hippocampal gyri (Figures 2C and 4C; Table 1). As shown in a wholebrain comparison of their extent against the extent of object activations (Figure 6), these Chinese words' activations were remarkably "object-like." They were bilateral (left hemisphere: $Z=3.40$; right hemisphere: $Z=3.16$ ) and symmetrical, and they included activations of the right-hemisphere lateral occipital area involved in object recognition (Grill-Spector \& Malach, 2004; Kanwisher, McDermott, \& Chun, 1997; MNI: 48 - 75 3), which is not activated by words in fluent readers (e.g., Szwed et al., 2011; Cohen et al., 2002). Such lateral occipital area activations were much less extensive in Chinese participants (Figure 6, arrows).

Finally, French participants did not show any activation for Chinese words over scrambled Chinese words in early or intermediate visual areas (Figures 2 and 4C; Table 1). This confirms that early and intermediate visual activations observed, respectively, only in French reading French and in Chinese reading Chinese, are dependent on the existence of reading expertise for a given script.

\section{Activations to French Nonwords}

When we contrasted French words to nonwords containing the very same letters as the words (see Methods and Figure 1), we found activations in language-related areas in the superior temporal gyri (STG) and STS in both French and Chinese participants (Table 1). This result confirmed that Chinese participants could at least partially recognize the French words that were presented. In addition, contrasting French words versus nonwords revealed activations in the anterior parts of the VWFA of French participants (MNI: $-39-39-21, Z=4.24$; below extent threshold), but not in Chinese participants, suggestive of French participants' having a more developed sensitivity to their native language orthographic structure in ventral occipitotemporal regions (Mano et al., 2013; Chan et al., 2009; Vinckier et al., 2007; Binder, Medler, Westbury, Liebenthal, \& Buchanan, 2006; Dehaene, Cohen, Sigman, \& Vinckier, 2005)

In early visual areas of French participants, activations to nonwords were higher than to scrambled words (lefthemisphere peak MNI: $-21-99-6, Z=3.80)$ and similar to words (no difference between the two stimuli, even at a very low threshold of $p<.05$ voxel-wise). This finding demonstrates that enhanced early visual activations to alphabetic stimuli are evoked by letter strings regardless of whether they form a word or not. 
Table 1. Summary of Main Activations for French Words, Chinese Words, and Objects in French and Chinese Participants

\begin{tabular}{|c|c|c|c|c|c|c|c|c|c|c|c|c|}
\hline \multirow{3}{*}{$\begin{array}{l}\text { Contrast } \\
\text { French words - scrambled }\end{array}$} & \multirow{3}{*}{$\frac{\text { Region }}{\text { Fusiform gyrus, BA } 37}$} & \multirow{3}{*}{$\frac{\text { Hemisphere }}{\mathrm{L}}$} & \multicolumn{5}{|c|}{ French Participants } & \multicolumn{5}{|c|}{ Chinese Participants } \\
\hline & & & \multirow{2}{*}{$\frac{Z \text { Score }}{7.01}$} & \multirow{2}{*}{$\begin{array}{c}\begin{array}{c}\text { Cluster } \\
\text { Size }\end{array} \\
1504\end{array}$} & \multicolumn{3}{|c|}{$\begin{array}{c}M N I \\
\text { Coordinates }\end{array}$} & \multirow{2}{*}{$\frac{Z \text { Score }}{-}$} & \multirow[t]{2}{*}{$\begin{array}{l}\text { Cluster } \\
\text { Size }\end{array}$} & \multicolumn{3}{|c|}{$\begin{array}{c}M N I \\
\text { Coordinates }\end{array}$} \\
\hline & & & & & -36 & -39 & -24 & & & & & \\
\hline & & & 5.95 & & -42 & -54 & -18 & 5.68 & 177 & -48 & -54 & -18 \\
\hline & & $\mathrm{R}$ & 3.1 & 573 & -57 & -48 & -12 & - & & & & \\
\hline & $\begin{array}{l}\text { Inferior occipital gyrus, } \\
\text { BA } 17 / 18\end{array}$ & $\mathrm{~L}$ & 3.43 & 471 & -9 & -93 & -3 & - & & & & \\
\hline & Middle frontal gyrus, & $\mathrm{L}$ & 6.45 & 1491 & -39 & 27 & 15 & 4.3 & 665 & -51 & 9 & 33 \\
\hline & & $\mathrm{R}$ & 5.1 & 1453 & 42 & 12 & 27 & 3.64 & 297 & 48 & 12 & 33 \\
\hline & Middle and superior & $\mathrm{L}$ & 6.3 & 1504 & -48 & -48 & 9 & - & & & & \\
\hline & temporal gyri & $\mathrm{R}$ & 4.94 & 573 & 48 & -39 & 6 & - & & & & \\
\hline & Superior parietal lobule, & $\mathrm{L}$ & 6.18 & 590 & -30 & -57 & 51 & 4.97 & 318 & -30 & -63 & 45 \\
\hline & $\mathrm{BA}$ & $\mathrm{R}$ & 5.03 & 398 & 33 & -57 & 48 & - & & & & \\
\hline \multirow[t]{12}{*}{ Chinese words - scrambled } & Fusiform gyrus, BA 37 & $\mathrm{~L}$ & 3.17 & 685 & -45 & -72 & -12 & $>8$ & 4687 & -45 & -51 & -18 \\
\hline & & $\mathrm{R}$ & 3.26 & 218 & 42 & -63 & -15 & 5.59 & 1587 & 45 & -51 & -21 \\
\hline & Middle occipital gyrus, & $\mathrm{L}$ & 3.47 & 685 & -42 & -81 & 0 & - & & & & \\
\hline & & $\mathrm{R}$ & 4.31 & 218 & 48 & -75 & -3 & - & & & & \\
\hline & $\begin{array}{l}\text { Inferior occipital gyrus, } \\
\text { BA 18/19 }\end{array}$ & $\mathrm{L}$ & - & & & & & 6.69 & 4687 & -45 & -84 & -6 \\
\hline & Parahippocampal gyrus & $\mathrm{L}$ & 3.88 & 685 & -30 & -30 & -6 & - & & & & \\
\hline & Middle frontal gyrus, & $\mathrm{L}$ & - & & & & & 6.67 & 4687 & -42 & 21 & 24 \\
\hline & & $\mathrm{R}$ & - & & & & & 5.8 & 1791 & 45 & 24 & 24 \\
\hline & Middle and superior & $\mathrm{L}$ & - & & & & & 4.56 & 4687 & -57 & -39 & 3 \\
\hline & temporal gyri & $\mathrm{R}$ & - & & & & & 4.01 & 1587 & 57 & -42 & 6 \\
\hline & Superior parietal lobule, & $\mathrm{L}$ & - & & & & & 7.35 & 4687 & -30 & -57 & 42 \\
\hline & BA 7 & $\mathrm{R}$ & - & & & & & 5.49 & 1587 & 39 & -63 & 51 \\
\hline \multirow[t]{3}{*}{ French words - nonwords } & Fusiform gyrus, BA 37 & $\mathrm{~L}$ & 4.24 & 192 & -39 & -39 & -21 & - & & & & \\
\hline & Middle and superior & $\mathrm{L}$ & 4.49 & 658 & -54 & -42 & 6 & 3.54 & 453 & -50 & -51 & 0 \\
\hline & temporal gyri & $\mathrm{R}$ & 3.37 & 375 & 45 & -39 & 6 & - & & & & \\
\hline \multirow[t]{4}{*}{ Objects - scrambled objects } & Fusiform gyrus, BA 37 & $\mathrm{~L}$ & $>8$ & 3379 & -48 & -75 & -3 & 6.62 & 2401 & -48 & -75 & -9 \\
\hline & & $\mathrm{R}$ & $>8$ & 3264 & 48 & -72 & -6 & $>8$ & 2440 & 45 & -51 & -18 \\
\hline & Middle frontal gyrus, & $\mathrm{L}$ & 3.69 & 245 & 39 & 12 & 24 & 3.84 & 263 & -45 & 3 & 30 \\
\hline & & $\mathrm{R}$ & 5.14 & 451 & 39 & 9 & 27 & 4.88 & 395 & 42 & 6 & 30 \\
\hline
\end{tabular}

\section{Effects of Attention, and Activation in the Attentional Network}

Words automatically elicit attention, and reading words evokes activations in the brain's attentional network. Top-down attention can also amplify activity in visual areas (e.g., Boynton, 2011; Silver, Ress, \& Heeger, 2007; Pessoa,
Kastner, \& Ungerleider, 2003; Kastner, Pinsk, De Weerd, Desimone, \& Ungerleider, 1999).

Could our results be explained by such an attentional amplification? Differences in attention allocation to different stimuli typically lead to differences in RTs. We found no significant participant group effects or interactions in RT data (Figure 1E). However, the detection times in 
Figure 4. Extent of activations to French and Chinese words. Activations induced by French words relative to scrambled French words (A, B, top row) and Chinese words relative to scrambled Chinese words (C, D, bottom row) in French participants (A, C, left column, $n=14$ ) and Chinese participants (B, D, right column, $n=11$ )

Enlargements (red boxes) show the left-hemisphere visual cortex. In both French (A) and Chinese (D) participants reading in their native script activated a similar network of language-related areas. In early visual areas, greater responses to words relative to scrambled controls were found only in French participants reading in their native script (white arrow and dotted circle), with no corresponding activations for other conditions/participant groups (white arrows). In intermediate visual areas, greater responses to words relative to scrambled controls were found only in Chinese readers reading in their native script (red arrow and solid circle), with no corresponding activations for other scripts/participant groups (red arrows). In Chinese participants, French words evoked activations stronger than scrambled words in left fusiform region. Results are overlaid on the average normalized T1 anatomy. Thresholds: $p<.01$ voxel-wise and $p<.05$ FDR corrected for multiple comparisons across the whole brain. the oddball task used here might not be relevant per se, as they do not directly reflect the time in which the word stimuli were read, but rather the amount of interference word stimuli had on detection of oddball targets.

Attentional amplification of visual activity should also be accompanied by activation of the frontoparietal areas, which drive attentional control (e.g., Pessoa et al., 2003; Kastner et al., 1999). We therefore examined activations related to attention in the brain's top-down attentional network. Relative to scrambled controls, all intact stimuli (Chinese words, French words, nonwords and objects) strongly activated the IPS (Table 1) and the FEF. We
Figure 5. Examples of intact French words versus scrambled French words contrast in two French participants. The images show unsmoothed data plotted on the individual participants' respective $\mathrm{T} 1$ anatomies. Thresholds: $p=.01$ voxel-wise and $p<.05$ FDR cluster-wise corrected for multiple comparisons across the whole brain.

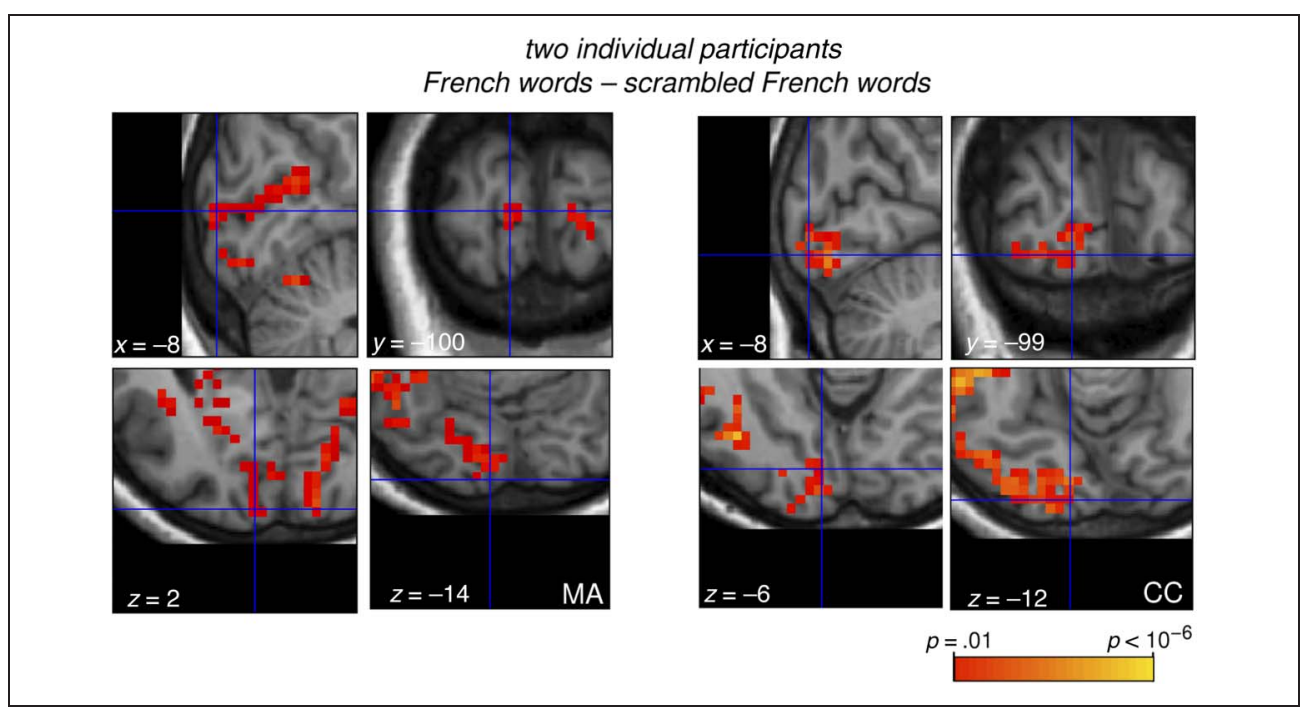


Figure 6. Activations to objects and Chinese words in French and Chinese participants. Activations to objects and Chinese words relative to scrambled controls in French participants (left column) and Chinese participants (right column). Objects usually activate both the lateral occipital and the fusiform parts of the ventral visual system (Grill-Spector \& Malach, 2004; Kanwisher et al., 1997), whereas words typically activate only the Fusiform area (Cohen et al., 2002). Here however, in French participants, Chinese words activated both the lateral occipital and the fusiform parts of the visual system (white arrows, left column). These "object-like" activations for Chinese words in area $\mathrm{LO}$ were much less extensive in Chinese participants (white arrows, right column). Results are overlaid on the average normalized T1 anatomy. Thresholds: $p<.01$ voxel-wise and $p<.05$ FDR corrected for multiple comparisons across the whole brain.

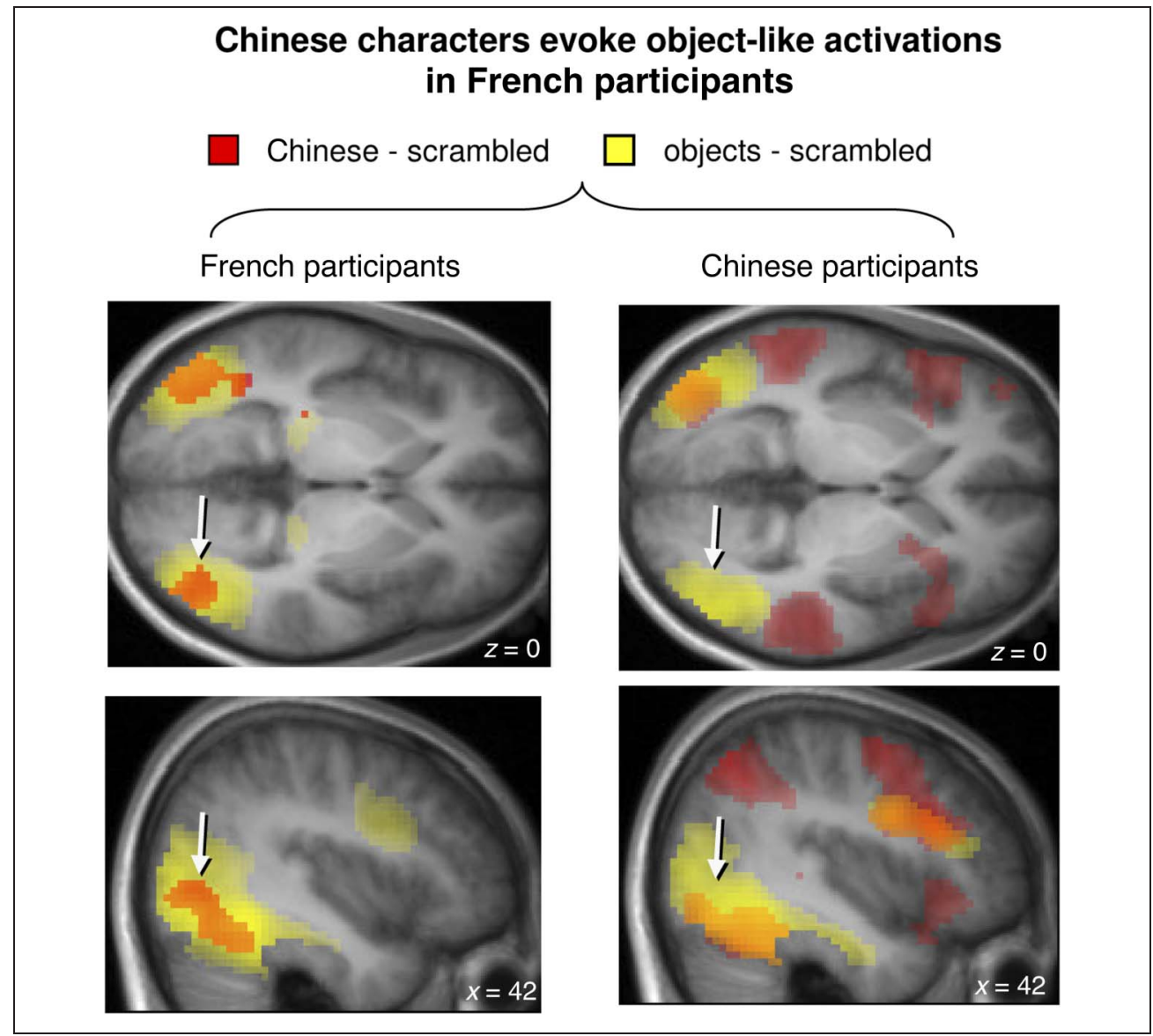

Figure 7. Activations to words and control stimuli in the attentional network (IPS). ROI analysis in individually localized participants' IPS (see Methods) revealed that Chinese participants showed similar responses to Chinese and French words, whereas French participants showed stronger weaker responses to Chinese than French words. The only significant differences between the two participants' populations were stronger activations for Chinese words in Chinese participants; French words evoked similar activations in both participants populations. $* * * p<.001$, $* * p<.01, * p<.05$. Activations in were computed relative to a fixation baseline. Error bars in represent the SEM across participants after subtraction of the individual participants' overall mean, equivalent to the error term in ANOVA with participants as random factor.

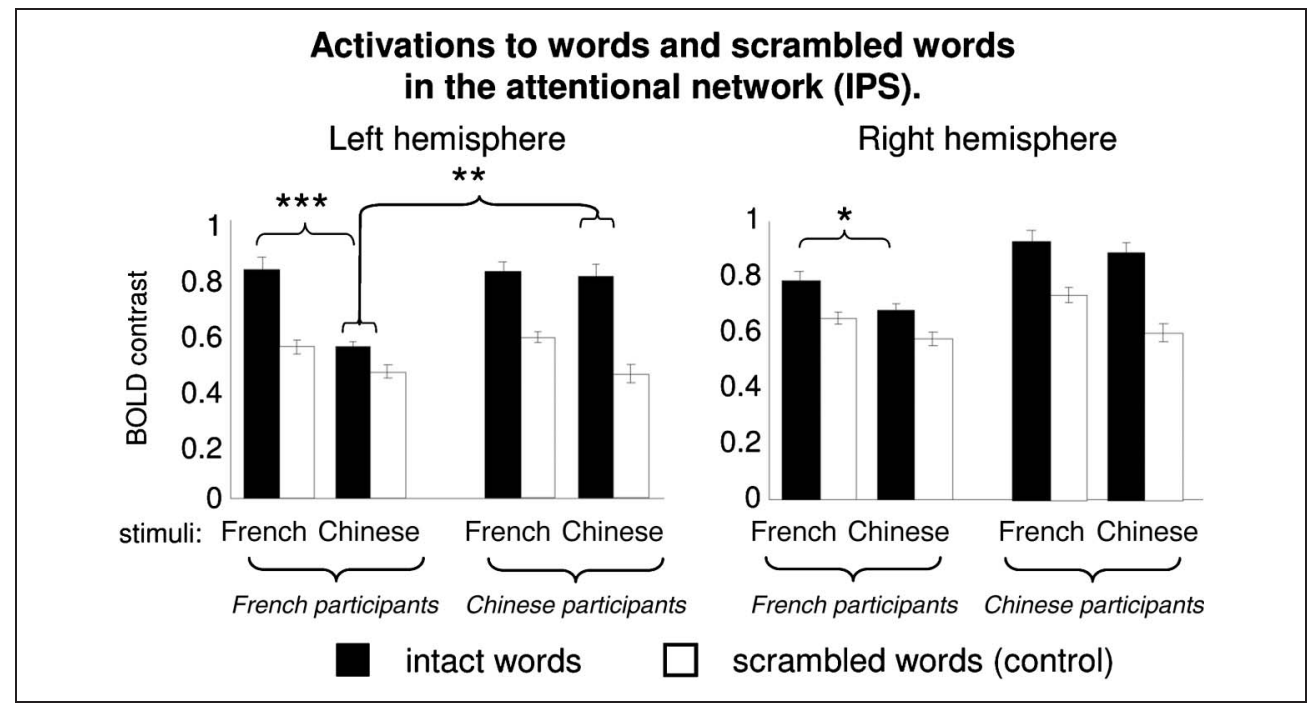


Figure 8. Whole-brain comparisons between French and Chinese participant groups during expert reading of their preferred script. (A) Regions that were activated more in the Chinese than in the French population by Chinese words relative to scrambled controls (orange-yellow). Regions more activated in the French population than in the Chinese population are shown in blue. (B) Regions that were activated in the French population more than in the Chinese population by French words relative to scrambled controls. (C) The differences between the neural networks used by two participant groups when reading in their native script (Chinese words_controls than in Chinese population vs. French words-controls in the French population). Results are overlaid on the average normalized T1 anatomy. Thresholds: $p<.01$ voxel-wise and $p<.05$ FDR corrected for multiple comparisons across the whole brain. examined these IPS activations in an ROI analysis (Figure 7; see Methods) and found (in addition to the general intact minus scrambled difference) that (1) French readers' IPS responses to Chinese words were weaker than their responses to French words (Figure $7 ; p=.001$, left hemisphere; $p=.03$, right hemisphere) and (2) Chinese readers responded similarly to both Chinese and French words ( $p>.26$ in both hemispheres). A comparison of the two groups' IPS activations showed that Chinese readers' responded stronger to Chinese words than French readers $(p=.02$; Hemisphere effects and Population $\times$ Hemisphere interactions, $p>3$ ) and that French words evoked similar activations in both participants' populations $(p=.36)$. Thus, the only significant differences between the two populations were that French participants, who were strictly monoliterate, had stronger activations in their familiar script, whereas the partially biliterate Chinese participants had similar activations for both scripts (Figure 7).

\section{Differences in Activation for Chinese and French Words in Chinese Participants}

Several differences have been reported between the reading networks for Chinese and Latin alphabets (Nakamura et al., 2012; Wu et al., 2012; Perfetti et al., 2010; Bolger et al., 2005; Tan et al., 2005). In this section, we analyze our Chinese participants' responses to words in both languages and examine whether our data are consistent with these earlier findings - with the qualification that our Chinese participants had only limited knowledge of French (Figure 1D). This section deals only with activations in the Chinese population.

When compared with the reading network for alphabetic script, the reading network for logographic script has been previously characterized by (1) larger activations in premotor areas (BA 9); (2) more symmetrical activations in the visual system, that is, stronger activations in the righthemisphere occipitotemporal regions; (3) larger activations in the left IPS; and (4) less activation of the left STS/STG (Wu et al., 2012; Perfetti et al., 2010; Bolger et al., 2005; Tan et al., 2005).

When we contrasted activations in Chinese participants reading Chinese versus activations in Chinese participants reading French words, we found that the largest and strongest region, which was more activated for Chinese words, was the left-hemisphere reading system encompassing the VWFA and more posterior visual areas (Figure $8 \mathrm{~A}$; peaks at MNI: $-42-48-21, Z=4.56$ and MNI: $-45-87-3, Z=3.99)$. More activation for Chinese than French words was also found in several areas often found in Chinese reading: the left premotor region (BA 9; 127 voxels, peak at MNI: $-42051, Z=3.69$ ), the righthemisphere visual system (208 voxels, peaks at MNI: $42-78-15, Z=3.47$ and MNI: $45-51-21, Z=3.51)$, and the IPS ( 58 voxels, peak at MNI: $-30-5339, Z=$ 3.29). Although these differences were not reliable against the conservative extent threshold used here, they fit well with previously published results (Perfetti et al., 2010; Tan et al., 2005).

\section{Comparisons between French and Chinese Participants}

We continued our exploration of the differences between the neural networks for reading in French and Chinese with a between-participant group analysis. In a whole-brain GLM analysis that included the participant group as a factor, we looked for differences in responses to French 
and Chinese words between the French and Chinese participants.

For the contrast of Chinese words minus scrambled controls, areas activated more strongly in Chinese than in French participants (Figure 8A, orange-yellow) included, first and foremost, the left VWFA (MNI: $-42-57-18$, $Z=7.06)$ and left-hemisphere intermediate visual cortex (MNI: $-39-81-9, Z=5.05$ ). Right-hemisphere fusiform differences were found only at a very exploratory threshold of $p=.025$ voxel-wise (MNI: $51-57-21$, $Z=2.52$, below extent threshold). Areas activated more strongly in French participants than in Chinese participants included the parahippocampal gyri (left hemisphere, MNI: $-30-36-12, Z=3.48$; right hemisphere, MNI: $30-39-12, Z=3.87$; Figure 8 A, blue) and the right lateral occipital area (MNI: $39-8712, Z=3.67$, below extent threshold).

For the contrast of French words minus scrambled controls, areas activated more strongly in French participants than in Chinese participants (Figure 8B) included, in particular, the left anterior fusiform gyrus (MNI: $-42-33$ $-21, Z=3.42$ ), with a small subpeak in the anterior VWFA (MNI: $-39-45-15, Z=2.98)$. There were no areas more activated in Chinese participants than French participants, even at an exploratory threshold of $p=.025$ voxel-wise.

Finally, we explored the differences between the neural networks, which the two participant groups used when reading in their native script (Figure $7 \mathrm{C}$; see Nakamura et al., 2012; Wu et al., 2012; Perfetti et al., 2010; Chan et al., 2009; Chen et al., 2009; Wong, Jobard, James, James, \& Gauthier, 2009; Bolger et al., 2005; Tan et al., 2005). Stronger activations in Chinese readers reading Chinese relative to French reading French were found in the lefthemisphere fusiform (MNI: $-39-63-21, Z=3.83$ ) and intermediate visual areas (MNI: $-39-81-6, Z=4.81$ ) and in the left inferior parietal sulcus (MNI: $-27-57$ $36, Z=4.24)$. The right-hemisphere fusiform difference was present (MNI: $45-57-21, Z=2.91$ ) but did not reach extent threshold. Importantly, we found no differences in BA 9 nor in the superior temporal regions, even below the usual extent threshold. Indeed, when we looked at BA 9 activations for ones' native language words versus scrambled controls in each participant group separately, they were similar in both groups (Chinese participants, Chinese words - scrambled MNI: - $422124, Z=$ 6.67; French participants, French words - scrambled MNI: $-421227, Z=7.28$ ). Finally, stronger activations in French readers reading French compared with Chinese readers reading Chinese were found only below the extent threshold, in the left STS/STG (105 voxels, MNI: $-45-45$ 12, $Z=3.07)$ and in the left IFG in BA 46 (245 voxels, MNI: $-363012, Z=-3.35)$.

\section{DISCUSSION}

Our main results can be summarized as follows.
First, the enhanced activations for alphabetic words relative to a scrambled control in early visual areas V1/ V2, previously observed by Szwed and colleagues (2011), were replicated and were found to be dependent on reading expertise in an alphabetic script (Figure 2). Indeed, Chinese participants, who were not expert French readers, did not show these activations when reading French words nor when reading Chinese words.

Second, for Chinese script, enhanced activations relative to scrambled controls in expert Chinese readers were observed in intermediate areas $\mathrm{V} 3 \mathrm{v} / \mathrm{hV} 4$ and not in early areas V1/V2 (Figure 2). Posterior to the VWFA, activations for Chinese script in Chinese readers (V3v/hV4) are thus topographically distinct from activations for Latin script in Latin readers (V1/V2).

\section{New Evidence for Expertise-dependent Involvement of Early Visual Areas in Reading}

In a previous study (Szwed et al., 2011) where participants saw French words and objects, we concluded that preferential processing for letter strings over control stimuli matched in line length, luminance, and number of line junctions exists not only in the high-level VWFA but also in early visual areas. In that study, we found that, unlike objects, words activated early visual areas stronger than scrambled controls. Nevertheless, it remained possible that these differences in responses to words and objects were due to some remaining low-level visual differences between these two classes of stimuli, as perfect matching of all visual attributes is almost impossible. Here, we circumvented that problem by using identical stimuli while varying the reading expertise of the participants.

Our experiment was a critical test for the role of early visual areas in reading expertise. The results replicated our previous findings: French participants exhibited stronger responses to French words than scrambled words in early visual areas. Chinese participants, who were not expert readers in French, failed to show this effect when reading French words. Since the stimuli, the task performance and the attention devoted to reading were exactly the same; the differences across groups must have come from differences in the participants' reading expertise. This result thus constitutes new evidence in favor of involvement of early visual areas in reading expertise.

The exact nature of this involvement remains to be explained. Previous studies indicate that extensive perceptual learning to detect an upright $\mathrm{T}$ shape among rotated distractors leads to behavioral improvement at specific retinal locations and to enhanced activation in area V1 (Sigman et al., 2005). Similarly, following extensive training, neurons in primate V1 can selectively enhance their responses to complex M- and V-like shapes (McManus, Li, \& Gilbert, 2011). It is therefore plausible that, with the acquisition of literacy in an alphabetic script, human V1 develops an improved encoding of letter shapes or letter fragments, which would at least partly account for the 
progressive increase in speed of reading in children (e.g., Grissemann, 1981; Lefavrais, 1967). There are several instances where expertise in a given faculty develops first in the high-order cortical areas, with expertise in earlier cortical areas following later on (e.g., Nahum, Daikhin, Lubin, Cohen, \& Ahissar, 2010; Ahissar, Nahum, Nelken, \& Hochstein, 2009).

Alternatively, those activations might not reflect local changes in the functional properties of area V1 but an expertise-dependent amplification related for instance to visual working memory (Harrison \& Tong, 2009; Serences, Ester, Vogel, \& Awh, 2009) or a process where early visual cortex acts as a "scratchpad" for increasing the precision of perceptual decisions (Chambers, Allen, Maizey, \& Williams, 2013; Lee, Mumford, Romero, \& Lamme, 1998). Arguably, such a perceptual decision-making process may be more prominent in Latin alphabet reading, where up to nine letters extending beyond the central field of view have to be read than during the perception of Chinese words comprising two to three foveal characters (see discussion of between-script differences below).

The enhanced activations that we observed in V1 were located to the central and horizontal parts of the retinotopic map. This could be expected, as French words are displayed horizontally and foveally. Actually, in left-to-right writing systems, the optimal gaze position for reading is left of words' center (Rayner, 1998), with most letters falling in the right half of the visual field. Accordingly, perceptual expertise, as indexed by reading accuracy, speed, and parallelism, predominates in the right hemifield (Nazir, Ben-Boutayab, Decoppet, Deutsch, \& Frost, 2004; Nazir, 2000). One may correlate this right visual field advantage with the fact that V1 specialization was observed in the left hemisphere (which contains mostly the right meridian representation) but not in the right hemisphere. This account would predict that in readers of right-to-left alphabets (such as Arabic or Hebrew) one should observe enhancement of responses to words in right-hemisphere V1, since right-to-left reading should call upon the left meridian representation (Lavidor, Alexander, \& McGraw, 2009; Lavidor \& Whitney, 2005; Nazir et al., 2004). Alternatively, the difference between left and right V1 might be explained by feedback from or colateralization with language areas, which are located in the left hemisphere in most of the population (Cai, Paulignan, Brysbaert, Ibarrola, \& Nazir, 2010; Pinel \& Dehaene, 2010). This explanation in turn would predict that, in participants whose language is lateralized to the right hemisphere (see e.g., Cai et al., 2010), one should see a corresponding enhancement of right V1 responses.

\section{Accommodation of Early and Intermediate Visual Areas to Specific Requirements of Chinese and Latin Scripts}

We found topographical differences in early and intermediate visual areas activated by the Latin alphabet (V1/ V2) versus Chinese characters (V3v/hV4).
The differences between the two scripts may reflect how the brain optimizes recognition by accommodating to specific properties of different scripts (Perfetti et al., 2010). Reading Chinese script poses a particular challenge to the visual system. Reading speed in words/minute is similar in French and Chinese, but skilled Chinese readers detect only two to three characters in one fixation (Inhoff \& Liu, 1998). Chinese characters have higher visual complexity, and each is made from a repertoire of a few hundred visual components called radicals. As explained in Perfetti et al. (2010),

the configuration of radicals can be left-right, topbottom, or inside-outside. The same two radicals, 口 and 木 can be combined to make the single-character words 杏 (apricot), 呆 (stupid), and 困 (sleepy), and the component radical 木 can also stand alone as the character for tree. More complex characters can be formed by vertically or horizontally inserting a radical between two others. [...] Component radicals often have their own meaning and pronunciation, and can provide cues for the meaning and pronunciation of the whole character. However, the low cue reliability of these radicals limits their general usefulness for character identification, making important whole character recognition processes.

Chinese radicals are also much more numerous (several hundreds) than the 26 Latin letters, and thus, each of them may not be seen repeatedly enough by a typical Chinese reader to support early-visual perceptual learning. Furthermore, Chinese phonetic radicals usually correspond to entire syllables, thus having a larger "phonological grain size" than letters, which usually map onto single phonemes (Ziegler \& Goswami, 2005).

We speculate that this mapping and the overall greater visual complexity of Chinese characters (see Methods) may go a long way toward explaining why their recognition places a greater emphasis on a relatively higher level of visual processing than the recognition of alphabetic characters. If the role of early and intermediate cortices in reading is one of a "scratchpad" (Chambers et al., 2013; Lee et al., 1998), then the V3v/hV4 "scratchpad" neurons have larger receptive fields (Zeki, 1993) and are capable of representing more complex features than in V1/V2 (e.g., Carlson, Rasquinha, Zhang, \& Connor, 2011). This would make them more suitable for representing more complex Chinese characters and combining them with a more complex phonological feedback (Ziegler \& Goswami, 2005). Similarly, if the enhanced response for intact words relative to scrambled controls is the result of perceptual learning, the greater complexity and variety of Chinese characters might result in perceptual learning taking place in $\mathrm{V} 3 \mathrm{v} / \mathrm{hV} 4$ rather than V1/V2. Our results thus show a flexible accommodation (Perfetti et al., 2010) or recycling (Dehaene \& Cohen, 2007) of the visual system to the slightly different challenges posed by these two scripts. 


\section{Feedback and Attention}

All the above-mentioned hypotheses allow for various degrees of contribution of top-down signals from higher visual and language areas. Current evidence points to a role for such signals in shaping functional response properties (e.g., Williams et al., 2008). The fact that we observed some enhancement of responses to French words over scrambled controls on the vertical meridian, where no stimuli were present (Figure 2D), can be cautiously interpreted as a signature of such feedback: Feedback projections to V1 from higher-order visual cortex are divergent and typically cover a broader retinotopic territory than the corresponding feedforward connections (e.g., Van Essen, 2004). Thus, V1 activations in a region that was not actually stimulated (i.e., on the vertical meridian; see Figure 2D) could be seen as a "footprint" of feedback activity. Because fMRI measurements might be particularly sensitive to top-down feedback (Boynton, 2011), future experiments should determine the role of feedback in the phenomena described here.

On the other hand, we believe that one of our results cannot be entirely explained by attentional effects. The "attentional spotlight" amplification of visual activity is accompanied by large activation in frontoparietal attentional areas themselves (e.g., Pessoa et al., 2003; Kastner et al., 1999). Our results (Figure 7) suggest that the same amount of attention was deployed in Chinese participants reading Chinese, Chinese participants reading French, and French participants reading French-and yet these conditions differed in their activation in V1/V2 and V3/V4. It is difficult to imagine how the same amount of attention could selectively amplify different visual areas (V1/V2 vs. V3/V4). Attentional amplification can be specific to a retinotopic location but seems unlikely to amplify one visual area (e.g., V1) while not amplifying another, neighboring one (e.g., V3; see, e.g., Silver et al., 2007; Pessoa et al., 2003). Moreover, this hypothetical amplification would have to occur only during reading in a native script; Chinese participants reading French activate their attentional network just as much as when they read Chinese, yet we see no enhancement of their visual response in early or intermediate areas.

\section{Comparison with Previous Results}

Dehaene and colleagues (2010) described a stronger response of $\mathrm{V} 1$ to horizontal checkers in literates relative to illiterates, whereas no such difference was found with vertical checkers (Dehaene et al., 2010). These findings are congruent with the enhancement of horizontal meridian response to words in the present study (Figure 2), although the bilaterally enhanced responses in V1/V2 to horizontal checkerboards reported by Dehaene and colleagues already in slowly reading ( $\sim 50$ words per minute) ex-illiterates might correspond to a different, more basic form of visual expertise than the one described here.
In a previous study (Szwed et al., 2011), we found that similar, enhanced activations to French words were the strongest in left hemisphere V1/V2, but also extended to left-hemisphere V3v/hV4 and right-hemisphere V1/ V2. In the current study, this enhancement was confined to the left-hemisphere V1/V2 (Figures 2, 4, and 5). This may be due to the differences in the word stimuli used. Duration (200 msec) and length (six to eight letters) were the same in both studies, but Szwed and colleagues (2011) used degraded words that were more challenging to recognize (80\% correct; Szwed et al., 2009, 2011). In contrast, the current experiment used easily recognizable intact words (100\% correct; Figure 1D). This discrepancy could be explained by an increase in early and intermediate visual processing in a situation where stimuli are more difficult and underspecified, and the role of Bayesian perceptual expectation mechanisms is increased (see Summerfield \& Koechlin, 2008).

\section{Neural Correlates of Fluent Reading}

Besides the observed differences in the visual system, our data offer mixed results regarding the claim that the reading network radically differ with culture (Wu et al., 2012; Perfetti et al., 2010; Bolger et al., 2005; Tan et al., 2005).

Several previous studies have reported that, in the visual system, Chinese reading networks are more symmetrical than those of readers of the Latin alphabet. A meta-analysis of six fMRI experiments in expert Chinese readers showed that, although the left-hemisphere VWFA is more extensively activated by reading than the corresponding right-hemisphere region (threefold difference in volume activated), that right-hemisphere region is, nonetheless, more active in Chinese readers (relative to similar nine studies in English and German readers, peak MNI: $34-62$-18; Tan et al., 2005). Another meta-analysis by Bolger and colleagues (2005) also found evidence for greater involvement of the right hemisphere in reading Chinese. In that second meta-analysis, the largest effect was located in more posterior, occipital regions; the VWFA of Chinese readers was found to be as left-lateralized as in English language readers.

However, other studies did not find such significant differences between reading in Chinese and Latin scripts. For example, Wong and colleagues (2009) studied a population of English-Chinese bilinguals and found that fusiform responses to both English and Chinese characters were strongly left-lateralized in both cases. Xue and colleagues (2005) found strong left-hemispheric dominance in 12-year-old Chinese children, and Chan and colleagues (2009) found that, in Chinese readers, only the left-hemisphere visual system shows a spatial hierarchical organization of brain responses to increasingly languagelike word forms, as occurs for Latin script readers (Vinckier et al., 2007; Binder et al., 2006).

Another major script-specific difference was reported in a lateral prefrontal region within BA 9, just anterior to left 
precentral cortex. There, some meta-analyses suggested a greater activation in Chinese compared with alphabetic readers (Wu et al., 2012; Bolger et al., 2005; Tan et al., 2005), perhaps due to great demands on addressed phonology (Tan et al., 2005) or visuospatial working memory (Wu et al., 2012). However, again, several other studies reported no statistically significant differences in BA 9 activation between Chinese and Latin alphabet readers (Nakamura et al., 2012; Sun, Yang, Desroches, Liu, \& Peng, 2011; Chee et al., 2000).

Our results provide mixed evidence on this participant. We did not find evidence for the putatively stronger involvement of BA 9 in Chinese reading. As to the differences in the right-hemisphere fusiform cortex, they were visible in fine-grained analyses based on individual ROIs (Figure 3) but not in whole-brain GLM analyses (Figure 8). Thus, whereas the Chinese readers' righthemisphere fusiform activations might be somewhat stronger, the left-hemisphere fusiform response remains predominant.

These left-hemisphere fusiform responses to Chinese characters in fluent Chinese readers can be contrasted to naive responses to Chinese characters in French readers who cannot read Chinese (Figure 6). The naive responses are equally strong in both hemispheres (Table 1) and include activations of lateral occipital object recognition areas that are not observed in fluent readers. Learning to read fluently is thus accompanied by a decrease of activity in the other parts of the ventral visual stream, which can be interpreted as a decreasing reliance on general form recognition mechanisms (see also discussion in Cai et al., 2010 and a similar effect for another type of visual expertise reported by Harley et al., 2009; Turkeltaub, Gareau, Flowers, Zeffiro, \& Eden, 2003; Shaywitz et al., 2002).

In summary, our study emphasizes both the cross-script invariance of the general circuitry for reading, which engages the VWFA in all scripts, and the small script-specific accommodations that occur in retinotopic cortex. In the future, it will be interesting to examine whether similar fine-grained adaptations can be found within auditory areas, which are also changed by reading (Dehaene et al., 2010). One may expect that, here again, the alterations in the neural code for spoken language induced by literacy are subtly different for phoneme- versus syllable-based writing systems (Cheung \& Chen, 2004; Cheung, Chen, Lai, Wong, \& Hills, 2001).

\section{Acknowledgments}

We would like to thank Séverine Roger, Séverine Desmidt, Chantal Ginsty, Véronique Joly, Bernadette Martins, Laurence Laurier, Gaelle Mediouini-Cloarec, Ghislaine Dehaene-Lambertz, Denis Le Bihan, Lucie Hertz-Pannier, Andreas Kleinschmidt, Caroline Huron, Sebastian Rodrigo, Eric Jouvent, and the rest of the LBIOM team of the NeuroSpin center for their help in participant recruitment and scanning and Chunxue Xia and Ran Zhang for special help in recruiting Chinese participants. We gratefully acknowledge Christophe Pallier for the code for making non- words out of words, Stephane Dufau for the code for measurement of stimulus perimetric compexity, and Dorota Kawecka for designing the objects made out of words. This research was funded by INSERM, CEA, Collège de France, University Paris XI, an Agence Nationale de Recherche (ANR, CORELEX project) grant to S. D. and L. C., and a "Sonata Bis" grant from the Narodowe Centrum Nauki to M. S. (2012/05/E/HS6/03538). M. S. was supported by an International Human Frontier Science Program Organisation Long-Term fellowship.

Reprint requests should be sent to Marcin Szwed, Department of Psychology, Jagiellonian University, Mickiewicza 3, 31-120 Kraków, Poland, or via e-mail: mfszwed@gmail.com.

\section{REFERENCES}

Ahissar, M., Nahum, M., Nelken, I., \& Hochstein, S. (2009). Reverse hierarchies and sensory learning. Philosophical Transactions of the Royal Society of London, Series B, Biological Sciences, 364, 285-299.

Ben-Shachar, M., Dougherty, R. F., Deutsch, G. K., \& Wandell, B. A. (2007). Differential sensitivity to words and shapes in ventral occipito-temporal cortex. Cerebral Cortex, 17, 1604-1611.

Binder, J. R., Medler, D. A., Westbury, C. F., Liebenthal, E., \& Buchanan, L. (2006). Tuning of the human left fusiform gyrus to sublexical orthographic structure. Neuroimage, 33, 739-748.

Bolger, D. J., Perfetti, C. A., \& Schneider, W. (2005). Cross-cultural effect on the brain revisited: Universal structures plus writing system variation. Human Brain Mapping, 25, 92-104.

Boynton, G. M. (2011). Spikes, BOLD, attention, and awareness: A comparison of electrophysiological and fMRI signals in V1. Journal of Vision, 11, 1-16.

Cai, Q., \& Brysbaert, M. (2010). SUBTLEX-CH: Chinese word and character frequencies based on film subtitles. PLoS One, 5, e10729.

Cai, Q., Paulignan, Y., Brysbaert, M., Ibarrola, D., \& Nazir, T. A. (2010). The left ventral occipito-temporal response to words depends on language lateralization but not on visual familiarity. Cerebral Cortex, 20, 1153-1163.

Carlson, E. T., Rasquinha, R. J., Zhang, K., \& Connor, C. E. (2011). A sparse object coding scheme in area V4. Current Biology, 21, 288-293.

Chambers, C. D., Allen, C. P., Maizey, L., \& Williams, M. A. (2013). Is delayed foveal feedback critical for extra-foveal perception? Cortex, 49, 327-335.

Chan, S. T., Tang, S. W., Tang, K. W., Lee, W. K., Lo, S. S., \& Kwong, K. K. (2009). Hierarchical coding of characters in the ventral and dorsal visual streams of Chinese language processing. Neuroimage, 48, 423-435.

Chee, M. W., Weekes, B., Lee, K. M., Soon, C. S., Schreiber, A., Hoon, J. J., et al. (2000). Overlap and dissociation of semantic processing of Chinese characters, English words, and pictures: Evidence from fMRI. Neuroimage, 12, 392-403.

Chen, C., Xue, G., Mei, L., Chen, C., \& Dong, Q. (2009). Cultural neurolinguistics. Progress in Brain Research, 178, 159-171.

Cheung, H., \& Chen, H. C. (2004). Early orthographic experience modifies both phonological awareness and on-line speech processing. Language and Cognitive Processes, 19, 1-28.

Cheung, H., Chen, H. C., Lai, C. Y., Wong, O. C., \& Hills, M. (2001). The development of phonological awareness: Effects of spoken language experience and orthography. Cognition, 81, 227-241. 
Claeys, K. G., Dupont, P., Cornette, L., Sunaert, S., Van Hecke, P., De Schutter, E., et al. (2004). Color discrimination involves ventral and dorsal stream visual areas. Cerebral Cortex, 14, 803-822.

Cohen, L., Lehericy, S., Chochon, F., Lemer, C., Rivaud, S., \& Dehaene, S. (2002). Language-specific tuning of visual cortex? Functional properties of the Visual Word Form Area. Brain, 125, 1054-1069.

Dehaene, S., \& Cohen, L. (2007). Cultural recycling of cortical maps. Neuron, 56, 384-398.

Dehaene, S., Cohen, L., Sigman, M., \& Vinckier, F. (2005). The neural code for written words: A proposal. Trends in Cognitive Sciences, 9, 335-341.

Dehaene, S., Pegado, F., Braga, L. W., Ventura, P., Filho, G. N., Jobert, A., et al. (2010). Impact of literacy on the cortical networks for vision and language. Science, 3, 1359-1364.

Grill-Spector, K., Kushnir, T., Hendler, T., \& Malach, R. (2000). The dynamics of object-selective activation correlate with recognition performance in humans. Nature Neuroscience, 3, 837-843.

Grill-Spector, K., \& Malach, R. (2004). The human visual cortex. Annual Review of Neuroscience, 27, 649-677.

Grissemann, H. (1981). Förderdiagnostik bei gestörtem Schriftspracherwerb. Bern: Huber Verlag.

Harley, E. M., Pope, W. B., Villablanca, J. P., Mumford, J., Suh, R., Mazziotta, J. C., et al. (2009). Engagement of fusiform cortex and disengagement of lateral occipital cortex in the acquisition of radiological expertise. Cerebral Cortex, 19, 2746-2754.

Harrison, S. A., \& Tong, F. (2009). Decoding reveals the contents of visual working memory in early visual areas. Nature, 458, 632-635.

Inhoff, A. W., \& Liu, W. (1998). The perceptual span and oculomotor activity during the reading of Chinese sentences. Journal of Experimental Psychology: Human Perception and Performance, 24, 20-34.

Kanwisher, N., McDermott, J., \& Chun, M. M. (1997). The fusiform face area: A module in human extrastriate cortex specialized for face perception. Journal of Neuroscience, 17, 4302-4311.

Kastner, S., Pinsk, M. A., De Weerd, P., Desimone, R., \& Ungerleider, L. G. (1999). Increased activity in human visual cortex during directed attention in the absence of visual stimulation. Neuron, 22, 751-761.

Lavidor, M., Alexander, T., \& McGraw, P. V. (2009). Word recognition processes modulate the naso-temporal asymmetry of the human visual field. Perception, 38 , 1536-1541.

Lavidor, M., \& Whitney, C. (2005). Word length effects in Hebrew. Brain Research, Cognitive Brain Research, 24, 127-132.

Lee, T. S., Mumford, D., Romero, R., \& Lamme, V. A. (1998). The role of the primary visual cortex in higher level vision. Vision Research, 38, 2429-2454.

Lefavrais, P. (1967). Test de l'Alouette Editions du Centre de Psychologie Appliquée. Paris: Clio Press.

Mano, Q. R., Humphries, C., Desai, R. H., Seidenberg, M. S., Osmon, D. C., Stengel, B. C., et al. (2013). The role of left occipitotemporal cortex in reading: Reconciling stimulus, task, and lexicality effects. Cerebral Cortex, 23, 988-1001.

Marinkovic, K., Dhond, R. P., Dale, A. M., Glessner, M., Carr, V., \& Halgren, E. (2003). Spatiotemporal dynamics of modality-specific and supramodal word processing. Neuron, 38, 487-497.

McManus, J. N., Li, W., \& Gilbert, C. D. (2011). Adaptive shape processing in primary visual cortex. Proceedings of the National Academy of Sciences, U.S.A., 108, 9739-9746.
Nahum, M., Daikhin, L., Lubin, Y., Cohen, Y., \& Ahissar, M. (2010). From comparison to classification: A cortical tool for boosting perception. Journal of Neuroscience, 30, $1128-1136$

Nakamura, K., Kuo, W. J., Pegado, F., Cohen, L., Tzeng, O. J., \& Dehaene, S. (2012). Reading by eye and reading by hand: Two universal brain systems for reading. Proceedings of the National Academy of Sciences, U.S.A., 109, 20762-20767.

Nazir, T. A. (2000). Traces of print along the visual pathway. In A. Kennedy, R. Radach, D. Heller, \& J. Pynte (Eds.), Reading as a perceptual process (pp. 3-22). Amsterdam: Elsevier.

Nazir, T. A., Ben-Boutayab, N., Decoppet, N., Deutsch, A., \& Frost, R. (2004). Reading habits, perceptual learning, and recognition of printed words. Brain and Language, 88, 294-311

New, B., Pallier, C., Brysbaert, M., \& Ferrand, L. (2004). Lexique 2: A new French lexical database. Behavior Research Methods, Instruments, \& Computers, 36, 516-524.

Pelli, D. G., Burns, C. W., Farell, B., \& Moore-Page, D. C. (2006). Feature detection and letter identification. Vision Research, 46, 4646-4674.

Perfetti, C. A., Nelson, J., Liu, Y., Fiez, J., \& Tan, L.-H. (2010). The neural bases of reading: Universals and writing system variations. In P. Cornelissen, M. Kringelbach, \& P. Hansen (Eds.), The neural basis of reading (pp. 147-172). Oxford: Oxford University Press.

Pessoa, L., Kastner, S., \& Ungerleider, L. G. (2003). Neuroimaging studies of attention: From modulation of sensory processing to top-down control. Journal of Neuroscience, 23, 3990-3998.

Pinel, P., \& Dehaene, S. (2010). Beyond hemispheric dominance: Brain regions underlying the joint lateralization of language and arithmetic to the left hemisphere. Journal of Cognitive Neuroscience, 22, 48-66.

Rayner, K. (1998). Eye movements in reading and information processing: 20 Years of research. Psychological Bulletin, 124, 372-422.

Schira, M. M., Wade, A. R., \& Tyler, C. W. (2007). Twodimensional mapping of the central and parafoveal visual field to human visual cortex. Journal of Neurophysiology, 97, 4284-4295.

Serences, J. T., Ester, E. F., Vogel, E. K., \& Awh, E. (2009). Stimulus-specific delay activity in human primary visual cortex. Psychological Science, 20, 207-214.

Shaywitz, B. A., Shaywitz, S. E., Pugh, K. R., Mencl, W. E., Fulbright, R. K., Skudlarski, P., et al. (2002). Disruption of posterior brain systems for reading in children with developmental dyslexia. Biological Psychiatry, 52, 101-110.

Sigman, M., Pan, H., Yang, Y., Stern, E., Silbersweig, D., \& Gilbert, C. D. (2005). Top-down reorganization of activity in the visual pathway after learning a shape identification task. Neuron, 46, 823-835.

Silver, M. A., Ress, D., \& Heeger, D. J. (2007). Neural correlates of sustained spatial attention in human early visual cortex. Journal of Neurophysiology, 97, 229-237.

Summerfield, C., \& Koechlin, E. (2008). A neural representation of prior information during perceptual inference. Neuron, 59, 336-347.

Sun, Y., Yang, Y., Desroches, A. S., Liu, L., \& Peng, D. (2011). The role of the ventral and dorsal pathways in reading Chinese characters and English words. Brain and Language, 119, 80-88.

Szwed, M., Cohen, L., Qiao, E., \& Dehaene, S. (2009). The role of invariant features in object and visual word recognition. Vision Research, 49, 718-725. 
Szwed, M., Dehaene, S., Kleinschmidt, A., Eger, E., Valabregue, R., Amadon, A., et al. (2011). Specialization for written words over objects in the visual cortex. Neuroimage, 56, 330-344.

Tan, L. H., Laird, A. R., Li, K., \& Fox, P. T. (2005). Neuroanatomical correlates of phonological processing of Chinese characters and alphabetic words: A meta-analysis. Human Brain Mapping, 25, 83-91.

Turkeltaub, P. E., Gareau, L., Flowers, D. L., Zeffiro, T. A., \& Eden, G. F. (2003). Development of neural mechanisms for reading. Nature Neuroscience, 6, 767-773.

Uchida, I., Kikyo, H., Nakajima, K., Konishi, S., Sekihara, K., \& Miyashita, Y. (1999). Activation of lateral extrastriate areas during orthographic processing of Japanese characters studied with fMRI. Neuroimage, 9, 208-215.

Van Essen, D. C. (2004). Organization of visual areas in macaque and human cerebral cortex. In L. M. Chalupa \& J. S. Werner (Eds.), The visual neurosciences (pp. 507-521) Cambridge, MA: MIT Press.

Vinckier, F., Dehaene, S., Jobert, A., Dubus, J. P., Sigman, M., \& Cohen, L. (2007). Hierarchical coding of letter strings in the ventral stream: Dissecting the inner organization of the visual word form system. Neuron, 55, 143-156.

Vul, E., Harris, C., Winkielman, P., \& Pashler, H. (2009). Puzzlingly high correlations in fMRI studies of emotion, personality, and social cognition. Perspectives on Psychological Science, 4, 274-290.

Williams, M. A., Baker, C. I., Op de Beeck, H. P., Shim, W. M., Dang, S., Triantafyllou, C., et al. (2008). Feedback of visual object information to foveal retinotopic cortex. Nature Neuroscience, 11, 1439-1445.

Winawer, J., Horiguchi, H., Sayres, R. A., Amano, K., \& Wandell, B. A. (2010). Mapping hV4 and ventral occipital cortex: The venous eclipse. Journal of Vision, 10, 1.

Wong, A. C., Jobard, G., James, K. H., James, T. W., \& Gauthier, I. (2009). Expertise with characters in alphabetic and nonalphabetic writing systems engage overlapping occipito-temporal areas. Cognitive Neuropsychology, 26, 111-127.

Wu, C. Y., Ho, M. H., \& Chen, S. H. (2012). A meta-analysis of fMRI studies on Chinese orthographic, phonological, and semantic processing. Neuroimage, 63, 381-391.

Xue, G., Dong, Q., Chen, K., Jin, Z., Chen, C., Zeng, Y., et al. (2005). Cerebral asymmetry in children when reading Chinese characters. Brain Research, Cognitive Brain Research, 24, 206-214.

Zeki, S. (1993). Vision of the brain. Hoboken, NJ: Wiley-Blackwell.

Ziegler, J. C., \& Goswami, U. (2005). Reading acquisition, developmental dyslexia, and skilled reading across languages: A psycholinguistic grain size theory. Psychological Bulletin, 131, 3-29. 